\title{
Improved Distributed Regional Location Management Scheme for Mobile IP
}

\author{
Wenchao Ma and Yuguang Fang \\ Department of Electrical \& Computer Engineering \\ University of Florida \\ mawench@ufl.edu and fang@ece.ufl.edu
}

\begin{abstract}
Abstruct-Mobile IP is the current standard supporting user mobility in wireless IP networks. Mobile IP regional registration is proposed by IETF to reduce the Mobile IP location update cost by making the registration localized. A distributed regional registration scheme is also introduced to choose the GFAs dynamically. In the distributed scheme, the signaling burden is evenly distributed among the network and the regional network boundary is dynamically adjusted according to the mobile user's up-to-date mobility and traffic load. In this paper, we develop a system model to analyze the distributed scheme performance. We also propose an improved distributed scheme which is called improved distributed regional location management scheme in our paper. The analytical results show that the dynamic scheme can reduce the Mobile IP mobility management cost effectively and the improved scheme can perform better.
\end{abstract}

\section{INTRODUCTION}

Mobile IP is the mobility-enabling protocol developed by the Internet Engineering Task Force (IETF) to support global mobility in IP networks ([1]). If a Mobile Host (MH) roams to a forcign subnetwork. according to the current Mobile IP protocol, the $\mathrm{MH}$ can obtain a new IP address (CoA) from a router (FA) in the visited network. The CoA of the $\mathrm{MH}$ will change from subnet to subnet.

However. Mobile IP is not a good solution for users with high mobility. Its mechanism requires every $\mathrm{MH}$ to update its new CoA to the Home Agent (HA) whenever the MH moves from one subnet to another. even though the $\mathrm{MH}$ dose not communicate with others while moving. The location update cost in Mobile IP can be excessive, especially for the mobile users with relatively high mobility and Iong distance to their HAs.

In order to reduce the registration signaling traffic, the Mobile IP regional registration is proposed in [2]. The protocol employs the FA hierarchy to localize the registration traffic. In this protocol, the HA registers the publicly routable address of the Gateway FA (GFA) and the MHs location update messages establish funnels in a regional network along the path from MHs to GFA. In the IETF regional registration scheme, the network architecture is centralized. It is not clear and usually hard to determine the regional network size. To overcome this shortcoming, Xie and Akyildiz proposed a distributed dynamic regional location management scheme for Mobile IP ([3]). In this scheme. the GFAs and the regional network size are adjusted based on the user's current traffic load and mobility information.
In this paper, we develop a system model to analyze the distributed scheme performance in a novel approach. We attempt to improve the distributed schemes by introducing the user state concept in our scheme. In this paper, we also prove that our new scheme can improve the distributed scheme performance further.

This paper is organized as follows. In section II, we introduce the distributed and improved schemes details. We develop a system model and derive the cost functions in section III. The scheme performances are demonstrated in section IV. Section $\mathrm{V}$ gives the conclusion.

\section{DISTRIBUTED SCHEMES AND THE IMPROVEMENT}

The drawback of the centralized system is the lack of flexibility. The regional network size under one GFA is difficult to determine since the user mobility and load vary from time to time. In [3], the authors introduced a distributed regional registration scheme. In the scheme, each FA can function either as an FA or a GFA. When an $\mathrm{MH}$ enters a new regional network, the first FA of the subnet the MH visits will be the GFA. So, the GFAs and regional networks are distributed and specific to every mobile user. The signaling traffic can be distributed among the network evenly. There are two variations of distributed scheme. One is fixed and another is dynamic. In the fixed version, the regional network size is fixed for a user. While, the regional network size can change based on the user's up-to-date mobility and traffic load in the dynamic scheme. how to compute the optimal regional network size can be found in [3].

Although the GFA can reduce the location update signaling traffic, it introduces additional packet delivery cost too. When packets are intercepted by the HA and tunneled to the user's current $\mathrm{COA}$. The GFA receives them and checks the visitors list, then forwards them to the current FA. In the fixed scheme, if the mobile user is in a subnet whose FA is not the GFA, the packets have to traverse additional nodes before reach the destination and the total signaling cost may exceed the basic Mobile IP scheme. This is proved in our following analysis. The dynamic scheme can change the regional work size according to the user's current information so that less traffic is generated than the fixed one. However, the dynamic version can also involve additional packet delivery cost because the user current FA is usually not the current regional network GFA for the user. 
In this paper, we try to improve the distributed scheme performance by incorporating the user state concept into it. We call our scheme the improved distributed regional location management scheme. The basic idea is similar with the distributed scheme, however, the $\mathrm{MH}$ is labelled active or idle. The MH is in idle state when no packet is received for a system predefined period. When the $\mathrm{MH}$ is in idle state, the improved scheme is exactly the same as the distributed scheme. The protocol descriptions of the distributed scheme is in [3]. If the $\mathrm{MH}$ receives a packet, it turns to active state. When the $\mathrm{MH}$ is in active state, it will update the up-to-date COA assigned by the current FA to the HA no matter what the pre-computed regional network size is. If the $\mathrm{MH}$ keeps in active state, it updates the new $\mathrm{COA}$ whenever it changes. When the $\mathrm{MH}$ receives no packet for a predefined period, it tums back to idle state. Since the MH keeps receiving packets in active state, it can piggyback the current $\mathrm{CoA}$ to one or $\mathrm{few}^{\prime}$ acknowledgement messages so that the location update cost can be neglected. In the improved scheme, the regional network size is fixed usually because the network size affects the scheme performance in a different way from the distributed scheme. We can see it later in this paper. When the $\mathrm{MH}$ is in active state, the MH's current GFA is the MH's serving FA when packets arrive, so that the improved scheme can reduce the packet delivery cost effectively without increasing the location update cost. In the following part, we analyze and compare all the above scheme performances.

\section{SYSTEM MODEI. AND ANALYSIS}

In this section, we develop an analytical model to derive the location update and packet delivery cost functions for the distributed and improved schemes. Just like in [3], we do not consider the periodic binding update costs that an $\mathrm{MH}$ sends to its HA or FAs to refresh their caches. In our analysis, we define the following notations.

1: The number of distinct subnets the $\mathrm{MH}$ visited during the period of the last packet arrival and the final location update just before it.

(3: The call-to-mobility ratio (CMR).

$K$ : The regional network size.

$i_{h}$ : The location update cost to the HA.

$U_{y}:$ The location update cost to the GFA.

$D_{1}$ : The packet delivery cost from the HA to FA.

$D_{y}$ : The packet delivery cost from GFA to FA.

Fig. I gives an example of the scheme operation. In this example. the $\mathrm{MH}$ rcgistered its $\mathrm{COA}$ in the subnet whose $\mathrm{FA}$ is $G F A_{1}$. After three movements $\left(M_{1}, M_{2}\right.$ and $\left.M_{3}\right)$, the $\mathrm{MH}$ is in the subnet whose $\mathrm{FA}$ is $F A_{3}$ and receives a packet from current GFA $\left(G F A_{1}\right)$, so $\beta=3$ under this situation. After further $i$ movements, the $\mathrm{MH}$ is in the subnet whose FA is $F A_{n}$. The MH current GFA is $G F A_{n}$. Depending on the regional network size, the $G F A_{n}$ might be $G F A_{1}$ or not. In Fig.l, we assume the $\mathrm{MH}$ receives second packets in subnet charged by $F A_{n}$ after $i$ movements. We try to compute the total signaling costs between the two consecutive packets arrivals.

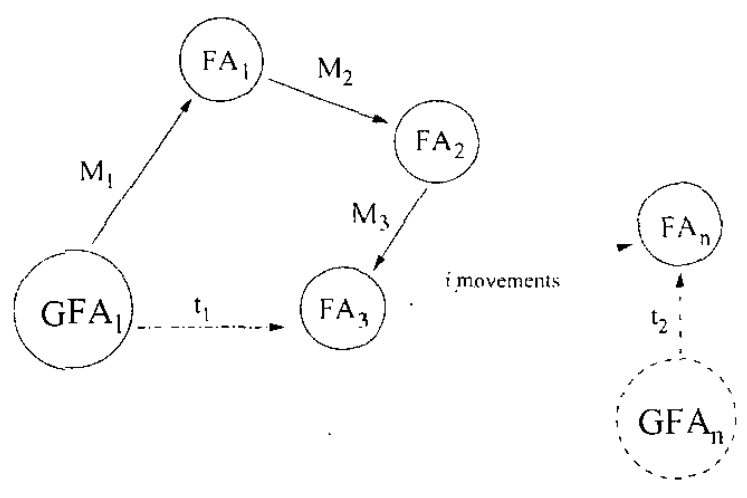

Fig. 1. The distributed Mobile if regional registration

Let $\alpha(i)$ denote the probability that an MH crosses $i$ subnets between two consecutive packet arrivals. Under the current Mobile IP scheme, the total signaling cost is ([7]):

$$
\begin{aligned}
C_{M I P} & =\sum_{i=0}^{\infty} i U_{h} a(i)+D_{h} \\
& =\frac{U_{h}}{\rho}+D_{h} .
\end{aligned}
$$

In this paper, the CMR is defined as follow: if packets arrive at an $\mathrm{MH}$ at rate $\lambda$ and the time the user resides in a given subnet has a mean $1 / \mu$, then the CMR, denoted by $\rho$, is given as $\rho=\lambda / \mu$. For the IETF regional registration protocol, if we assume the regional network size is $K$ and $i$ subnets are crossed between two packet arrivals, the $\mathrm{MH}$ will update to the $\mathrm{HA}\left\lfloor\frac{i+\beta}{K}\right\rfloor$ times and update the GFAs in the rest $i-\left\lfloor\frac{i+\beta}{K}\right\rfloor$ times. So the average location update cost function for the scheme can be written as

$$
C_{u}(\beta)=\sum_{i=0}^{\infty}\left(\left\lfloor\frac{i+\beta}{K}\right\rfloor U_{h}+\left(i-\left\lfloor\frac{i+\beta}{K}\right\rfloor\right) U_{g}\right) \alpha(i) .
$$

In the IETF scheme, the GFA might introduce additional cost besides the basic packet delivery cost. We assume that $\beta$ is uniformly distributed in our paper. So, when $i=0$, the additional cost generated by GFA is $\frac{K-1}{K} D_{g} \alpha(0)$. When $i>0$, no additional cost involves when $\frac{i+\beta}{K}=\left\lfloor\frac{i+\beta}{K}\right\rfloor$. Then the packet delivery cost is

$$
\begin{aligned}
C_{d}(\beta)= & D_{h}+\frac{K-1}{K} D_{g} \alpha(0)+\sum_{i=1}^{\infty} D_{g} \alpha(i) \\
& -\sum_{i+\beta=\left\lfloor\frac{i+k i+k}{\hbar}\right\rfloor K}^{\infty} D_{g} \alpha(i) .
\end{aligned}
$$

So, we have

$$
C_{D R M I P}(\beta)=C_{u}(\beta)+C_{d}(\beta) .
$$

For improved scheme, $\beta$ is zero all the time. So we can get the location update and packet delivery cost functions respectively 
in a similar way.

$$
\begin{aligned}
& C_{u}^{\prime}=\sum_{i=0}^{\infty}\left(\left\lfloor\frac{i}{K}\right\rfloor U_{h}+\left(i-\left\lfloor\frac{i}{K}\right\rfloor\right) U_{g}\right) \alpha(i), \\
& C_{d}^{\prime}=D_{h}+\sum_{i=0}^{\infty} D_{g} \alpha(i)-\sum_{i=\left\lfloor\frac{i}{K}\right\rfloor K}^{\infty} D_{g} \alpha(i) .
\end{aligned}
$$

Then.

$$
C_{\text {DRM/P }}^{\prime}=C_{u}^{\prime}+C_{d}^{\prime}
$$

In order to analyze the performances of the schemes, we need to evaluate $\alpha(i)$ in more detail. We assume the packet arrivals to an $\mathrm{MH}$ form a Poisson process with arrival rate $\lambda$ and the residence time of an $\mathrm{MH}$ in a subnet is a random variable with a general density function $f_{m}(t)$ and Laplace transform $f_{m}^{*}(s)=\int_{t=0}^{\infty} f_{m}(t) e^{-s t} d t$. For convenience, we denote $g=f_{m}^{*}(s)$. With the above assumption, $\alpha(i)$ is given by $([6])$

$$
\alpha(i)= \begin{cases}1-\frac{1-g}{\rho} & \text { if } i=0 \\ \frac{(1-g)^{2} g^{\prime-1}}{\rho} & \text { if } i>0\end{cases}
$$

We assume that $i=j k+q$, then

$$
a(j K+q)=\frac{(1-g)^{2}}{\rho g}\left(g^{K}\right)^{j} g^{q}=y z^{j} x^{q}
$$

where

$$
y=\frac{(1-g)^{2}}{\rho g} . \quad z=g^{K}, \quad x=y .
$$

Notice that both $0 \leq q<K$ and $0 \leq \beta<K$, we can rewrite (2) as

$$
\begin{aligned}
C_{u}(\beta)= & U_{g} \sum_{i=0}^{\infty} i \alpha(i)+\left(U_{h}-U_{g}\right) \sum_{i=0}^{\infty}\left[\frac{i+\beta}{K}\right\rfloor \alpha(i) \\
= & \frac{U_{g}}{\rho}+\left(U_{h}-U_{g}\right) \sum_{j=0}^{\infty} \sum_{q=0}^{K-1}\left\lfloor\frac{j K+q+\beta}{K}\right] y z^{j} x^{q} \\
= & \frac{U_{g}}{\rho}+\left(U_{h}-U_{g}\right) y \\
& \cdot \sum_{j=0}^{\infty}\left[j j^{j} \sum_{q=0}^{K-i j-1} x^{q}+(j+1) z^{j} \sum_{q=K-\beta}^{K-1+\beta} x^{q}\right] \\
= & \frac{U_{g}}{\rho}+\frac{\left(U_{h}-U_{g}\right)(1-g) g^{K-1}}{\rho\left(1-g^{K}\right)} \\
& \left.\cdot \frac{1-g^{K+\beta}}{1-g^{K}}+\frac{1-g^{2 \beta}}{g^{j}}\right] .
\end{aligned}
$$

In order to simplify (3), we assume $i+\beta=j K$, where $j=$ 1.2. .... . Then we have

$$
\begin{aligned}
C_{d}(\beta)= & D_{h}+\frac{K-1}{K} D_{g} \alpha(0)+\sum_{i=1}^{\infty} D_{g} \alpha(i) \\
& -\sum_{i+\beta=\left\lfloor\frac{i+i j}{\hbar}\right\rfloor K}^{\infty} D_{g} \alpha(i)
\end{aligned}
$$

$$
\begin{aligned}
= & D_{h}+\frac{(K-1) \rho+1-g}{K} \cdot \frac{D_{g}}{\rho} \\
& -\sum_{j=1}^{\infty} D_{g} \alpha(j K-\beta) \\
= & D_{h}+\frac{D_{g}}{\rho}\left[\frac{(K-1) \rho+1-g}{K}\right. \\
& \left.-\frac{(1-g)^{2} g^{K-\beta-1}}{1-g^{K}}\right] .
\end{aligned}
$$

If the $\beta$ is uniform distribution, we can have (4) like

$$
\begin{aligned}
C_{D R M I P}= & \frac{1}{K} \sum_{\beta=0}^{K-1} C_{D R M I P}(\beta) \\
= & \frac{U_{g}}{\rho}+D_{h}+\frac{K-1}{K} D_{g}+\frac{\left(U_{h}-\frac{\left.U_{g}\right)}{\rho} g^{K-1}\right.}{\rho} \\
& \cdot\left[\frac{1-g}{\left(1-g^{K}\right)^{2}}+\frac{g^{1-K}-g-1}{K\left(1-g^{K}\right)}\right]
\end{aligned}
$$

If we further assume the user residence time in a subnet is exponentially distributed, we have $g=\frac{1}{1+\rho}$, then (11) becomes

$$
\begin{aligned}
C_{D R M I P}= & \frac{U_{g}}{\rho}+D_{h}+\frac{K-1}{K} D_{g}+\left(U_{h}-U_{g}\right) \\
& \cdot\left[\frac{(1+\rho)^{K}}{\left[(1+\rho)^{K}-1\right]^{2}}+\frac{(1+\rho)^{K}-2-\rho}{K \rho\left[(1+\rho)^{K}-1\right]}\right] .
\end{aligned}
$$

Equation (12) is the cost function for IETF regional registration scheme. If the regional network size $K$ is replaced by the optimal values that can minimize the total signaling cost, then it becomes the cost function for dynamic scheme. How to get the optimal values can be found in [3].

We can also derive $C_{u}^{\prime}$ and $C_{d}^{\prime}$ in the similar way,

$$
\begin{aligned}
C_{u}^{\prime} & =U_{g} \sum_{i=0}^{\infty} i \alpha(i)+\left(U_{h}-U_{g}\right) \sum_{i=0}^{\infty}\left\lfloor\frac{i}{K}\right\rfloor \alpha(i) \\
& =\frac{U_{g}}{\rho}+\frac{\left(U_{h}-U_{g}\right)(1-g) g^{K-1}}{\rho\left(1-g^{K}\right)}
\end{aligned}
$$

Let we assume $i=j K$, where $j=0,1,2, \cdots, \infty, C_{d}^{\prime}$ can be shown as

$$
\begin{aligned}
C_{d}^{\prime} & =D_{h}+D_{g}-D_{g}\left(1-\frac{1-g}{\rho}\right)-D_{g} \sum_{j=1}^{\infty} \frac{(1-g)^{2} g^{j K}}{\rho g} \\
& =D_{h}+\frac{(1-g)\left(1-g^{K-1}\right) D_{g}}{\rho\left(1-g^{K}\right)}
\end{aligned}
$$

When $g=\frac{1}{1+\rho}$, we have (7) as

$C_{D R M I P}^{\prime}=\frac{U_{g}}{\rho}+D_{h}+\frac{U_{h}-U_{g}+\left[(1+\rho)^{K-1}-1\right] D_{g}}{(1+\rho)^{K}-1}$. 
TABLE I

PERFORMANCE ANALYSIS PARAMETERS

\begin{tabular}{|c|c|c|c|c|}
\hline$t_{h g}$ & $t_{g f}$ & $p_{h}$ & $p_{g}$ & $p_{f}$ \\
100 & 30 & 30 & 15 & 5 \\
\hline$m_{h g}$ & $m_{g f}$ & $h_{h}$ & $h_{f}$ & $\xi$ \\
50 & 15 & 20 & 1 & 0.1 \\
\hline
\end{tabular}

\section{NUMERICAL RESULTS}

In the following, we demonstrate the scheme performances under various conditions. We define the following notations for our analysis.

$t_{h g}$ : The location update transmission cost between HA and GFA.

$t_{g f}:$ The location update transmission cost between GFA and FA.

$p_{h}$ : The location update processing costs at HA.

$p_{g}$ : The location update processing costs at GFA.

$p_{f}$ : The location update processing costs at FA.

$m_{h g}$ : The packet delivery transmission cost between HA and GFA.

$m_{g f}$ : The packet delivery transmission cost between GFA and FA.

$h_{h}$ : The packet delivery processing cost in HA.

$h_{g}$ : The packet delivery processing cost in GFA.

According to the protocols and above definitions, we have

$$
\begin{aligned}
U_{h} & =2 t_{h g}+p_{h}+2 p_{g}, \\
U_{g} & =2 t_{g f}+p_{g}+2 p_{f}, \\
D_{h} & =m_{h g}+h_{h}+h_{g}, \\
D_{g} & =m_{g f}+h_{g}+h_{f} .
\end{aligned}
$$

In the above formula, we neglect the costs that are common to all the procedures, like the wireless channel transmission cost. The GFA processing cost is proportional to the number of subnets under it. Since IP routing table lookup is based on the longest prefix matching, then the GFA processing complexity is proportional to the logarithm of $K$. According to the above assumptions, we get $h_{g}=\xi K \log (K)$, where $\xi$ is the cost coefficient. In our analysis, we use the parameters listed in Table I for comparison.

Fig. 2 shows the total signaling costs for all the schemes under different call-to-mobility ratio (CMR). In this figure, when the CMR is small, the basic Mobile IP scheme generates the most signaling traffic. With the increase of CMR, the distributed scheme with fixed regional network size can generate more cost than the Mobile IP scheme does. The reason is that when the CMR is large, many packets have to go through GFA before reach the user's current FA. This procedure would introduce additional cost. The dynamic scheme results in less traffic than the fixed one dose and the total cost will never exceed the Mobile IP scheme. It is obvious that our improved scheme generates the least traffic all the time. In Fig.3, we show the relative total signaling costs of the three scheme

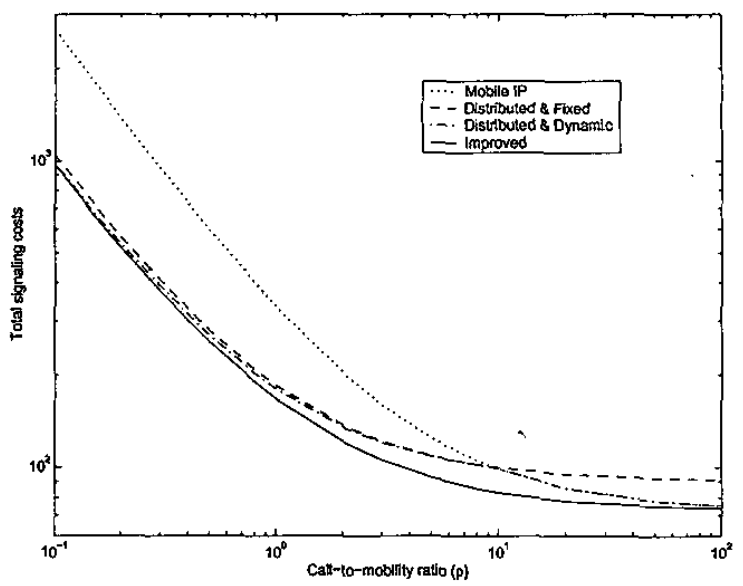

Fig. 2. Comparison of the total signaling cost

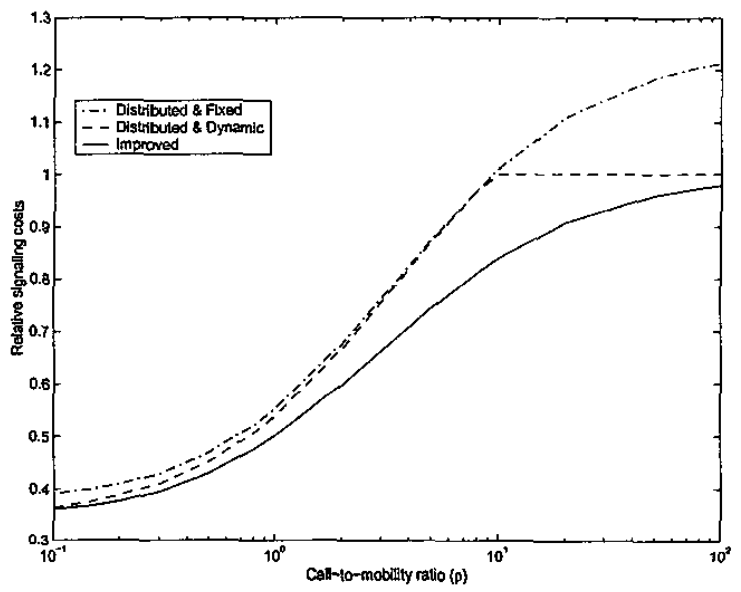

Fig. 3. Comparison of the relative signaling cost

to the Mobile IP scheme. In fact, when the CMR increases, the optimal values for the dynamic scheme decrease. If the optimal values become one, the dynamic scheme reduces to Mobile IP scheme. That is why the relative cost curve for dynamic scheme levels off when the CMR is large. The cost curve shows that the improved scheme outperforms both the fixed and dynamic schemes.

Now, we investigate how the regional network size can affect the scheme performances. In Fig. 4 and Fig.5, we vary the regional network size $K$ under different $C M R$ for the fixed scheme and the improved scheme. In Fig.4, we can see that when the regional network size is large, the fixed scheme can generate less traffic with large $K$. Since the GFA processing cost is proportional to the regional work size and the MHs number under it. The total cost for the fixed scheme increases fast with the CMR when the $K$ is large. From the figure we can conclude that it is impossible to find an optimal fixed regional network size for all the users under different traffic 


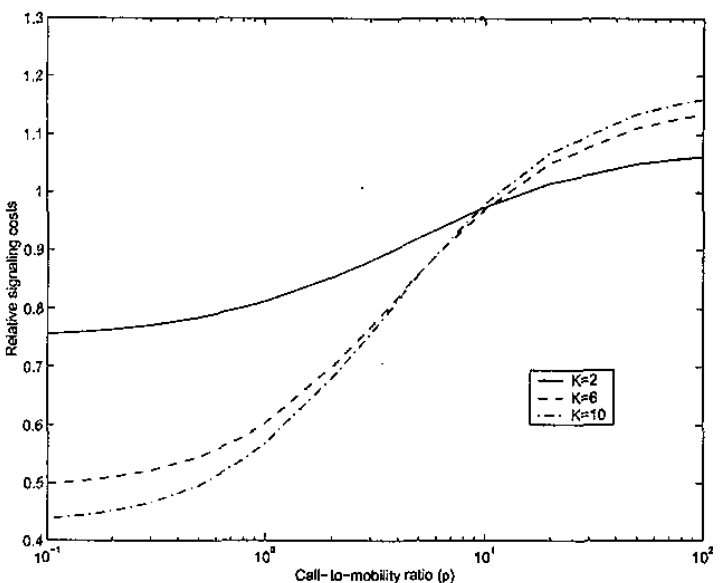

Fig. 4. Regional network size effect on the fixed scheme

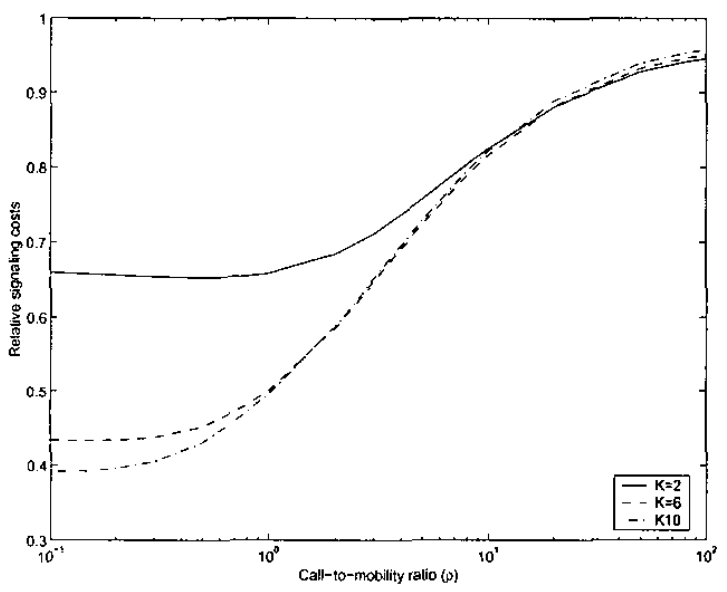

Fig. 5. Regional network size effect on the improved scheme

loads and mobilities. For the improved scheme (Fig.5), the regional network size has little effect on performance when the CMR is large. When the CMR is small, large $K$ can generate less system traffic load. However, lager $K$ can also aggravate the GFA burden, so the total cost saving diminishes with the increase of $K$.

\section{CONCLUSion}

In this paper, we introduced the distributed regional location management schemes for Mobile IP networks. We also proposed a new scheme to improve the distributed scheme performance which is called improved distributed scheme in the paper. In our works, we developed a model to derive the cost functions for all the schemes and compared their performances under different conditions. In our analysis, we assume the mobile user never visits a same subnet twice. In reality, a mobile user may move among subnets back and forth. So, the effective subnet number a user crossed between two consecutive packet arrivals is just a portion of the total subnet number crossed. This will not affect our conclusion. Based on our analysis, we conclude that the fixed scheme can have saving only when the CMR is small and the dynamic scheme has better performance than the fixed one and will never generate more traffic load than the basic Mobile IP scheme does. Our analytical results also show that the improved distributed scheme outperforms both the fixed and dynamic schemes under various situations.

\section{ACKNOWLEDGMENT}

This work was supported in part by National Science Foundation Faculty Early Career Development Award under grant ANI-0093241.

\section{REFERENCES}

[1] C. Perkins, "IP mobility support", Internet RFC 2002, October 1996. Work in progress.

[2] Eva Gustafsson, Annika Jonsson and Charles Perkins, "Mobile IP regional registration", Internet draft, draft-ietf-mobileip-reg-tunnel-04.txt, work in progress, March 2001.

[3] Jiang Xie and Ian F. Akyildiz, "A distributed dynamic regional locaion management scheme for mobile IP", Proc. IEEE INFOCOM 2002, pp. 1069-1078, 2002.

[4] C. Perkins and K-Y. Wang, "Optimized smooth handoffs in Mobile IP", Proceedings of IEEE Symposium on Computers and Communications, Egypt, July 1999.

[5] Wenchao Ma and Yuguang Fang, "Location Management Employing Two-Level Forwarding Pointers in PCS Networks", Proc. IEEE Globecom' 02 , Taipei, November 2002.

[6] Y. Fang, 1. Chlamtac, and Y.B. Lin. "Portable movement modeling for PCS networks", IEEE Trans. Veh.Technol., vol.87, no.8, pp.1347-1384, August 1999.

[7] R. Jain and Y.B. Lin, "An auxiliary user location strategy employing forwarding pointers to reduce network impacts of PCS". Wireless Networks 1, pp.197-210, 1995. 